
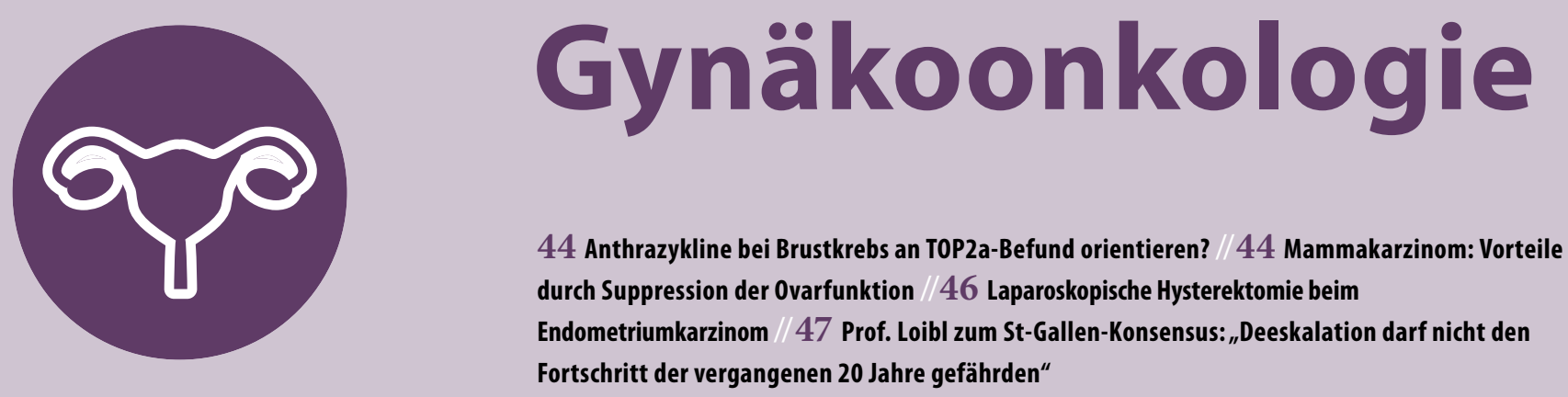

\title{
Anthrazykline bei Brustkrebs an TOP2a-Befund orientieren?
}

Inzwischen belegen Analysen aus einer ganzen Reihe hochrangiger Studien, dass Anthrazykline vor allem bei Veränderungen im Gen für die Topoisomerase 2a (TOP2a) effektiv sind, nicht aber bei normaler TOP2a. Das konnte jetzt auch durch eine Interventionsstudie bestätigt werden.

D ie Phase-III-Studie umfasste 2.012 Frauen mit einem frühen TOP2anormalen invasiven Mammakarzinom und mindestens einem Hochrisikofaktor (Alter $<39$ Jahre, Tumor $>20 \mathrm{~mm}$, duktales Karzinom vom Grad 2/3, Östrogenrezeptor(ER)-negativ und/oder HER2-positiv). Postoperativ erhielten sie randomisiert eine adjuvante Therapie mit 3 Zyklen Epirubicin/Cyclophosphamid, gefolgt von 3 Zyklen Docetaxel (EC-D) oder mit 6 Zyklen Docetaxel/Cyclophosphamid (DC). Nach median 69 Monaten war die 5-Jahres-Rate für das krankheitsfreie Überleben (DFS), den primären Endpunkt der Studie, mit 87,9\% im EC-D- und 88,3\% im anthrazyklinfreien Arm vergleichbar hoch. Einen signifikanten Unterschied in der Zahl der DFS-Ereignisse gab es in der Intention-to-treat-Analyse nicht (Hazard Ratio [HR] 1,00; $\mathrm{p}=1,00)$. Das galt auch bezüglich des fernmetastasenfreien Überlebens (HR 1,12; $\mathrm{p}=0,40$ ) und der Mortalität (HR 1,15; p = 0,41).

Es zeigte sich eine signifikante Interaktion des Menopausenstatus mit dem DFS $(p=0,04)$, nicht aber mit dem Gesamtüberleben (OS; $p=0,07)$ : Postmenopausale Frauen profitierten hinsichtlich des DFS von EC-D, prämenopausale Patientinnen dagegen von DC. Patientinnen mit einem Grad-3-Tumor hatten hinsichtlich des PFS sowie des OS bessere Ergebnisse mit DC, solche mit Grad1- und -2-Tumor mit EC-D.
Das anthazyklinhaltige Regime ging mit deutlich mehr nicht-hämatologischen Nebenwirkungen wie Stomatitis, Myalgien und Arthralgien, Übelkeit und Erbrechen, Fatigue sowie peripheren Neuropathien einher. Nur Ödeme waren unter dem DC-Regime häufiger.

Fazit: Die Studie ergänzt die Evidenz dafür, dass eine adjuvante Therapie mit An-

\section{An den Ergebnissen zweier Studien überprüften US-Onkologen den Nutzen einer Suppression der Ovari- alfunktion bei chemotherapierten jungen Frauen mit Hormonrezeptor(HR)-positivem Brustkrebs nach Resektion.}

$\mathrm{n}$ den Studien TEXT (Tamoxifen and Exemestane Trial) und SOFT (Suppression of Ovarian Function Trial) war geprüft worden, ob adjuvantes Exemestan der bisherigen Standardtherapie mit Tamoxifen bei Frauen mit HR-positivem Brustkrebs überlegen ist. Im Vergleich zur Tamoxifen-Therapie wurde das Rezidivrisiko mit Exemestan signifikant thrazyklinen beim frühen TOP2a-normalen Brustkrebs mit erhöhtem Risiko keinen Überlebensvorteil bietet und mit mehr Nebenwirkungen einhergeht. Die Interaktionsanalysen zeigen aber, dass das womöglich nicht für alle Patientinnen mit TOP2a-normalem Mammakarzinom gilt. Zudem weisen die Forscher darauf hin, dass die TOP2a-Tests derzeit nicht immer vergleichbare und reproduzierbare Ergebnisse liefern. Friederike Klein

Ejlertsen B et al. Adjuvant Cyclophosphamide and Docetaxel With or Without Epirubicin for Early TOP2A-Normal Breast Cancer: DBCG 07READ, an Open-Label, Phase III, Randomized Trial. J Clin Oncol. 2017;35(23):2639-46.

\section{Mammakarzinom: Vorteile durch Suppression der Ovarfunktion}

verringert. Unklar war bislang der genaue Stellenwert der ovariellen Suppression (OFS, „ovarian function suppression“). An den beiden Studien hatten insgesamt fast 6.000 prämenopausale Frauen teilgenommen.

Poornima Saha und Kollegen überprüften nun anhand der Studienergebnisse die Wirksamkeit der adjuvanten Therapie in Kombination mit einer OFS (mit dem GnRH-Agonisten Triptorelin, beidseitiger Oophorektomie oder Bestrahlung der Ovarien) und deren Einfluss auf die Lebensqualität. Eine optionale Chemotherapie wurde gleichzeitig mit Triptorelin gegeben. In beiden Studien zusammen waren fast 600 Patientinnen jünger als 35 Jahre. 